\title{
Diagnosis of Foregut and Tailgut Cysts by Endosonographically Guided Fine-Needle Aspiration
}

Diane A. Hall, M.D., Ph.D., Robert T. Pu, M.D., Ph.D., and Yijun Pang, M.D., Ph.D.

Foregut, hindgut, and tailgut cysts are uncommon developmental anomalies. Clinical and radiological diagnosis can present many challenges, especially in adult patients or when the lesions are in unique locations. Thus, diagnosis has traditionally been provided upon surgical resection. We describe the diagnoses of a gastric foregut cyst and a retrorectal tailgut cyst by endosonographically guided fine-needle aspiration in two adults. The common cytologic features of the specimens are ciliated epithelial cells, proteinaceous material with degenerated debris, histiocytes, and benign appearing epithelium of squamous and/or gastrointestinal type that lack cytologic atypia. The identification of ciliated columnar cells is the key finding. Cytologic diagnosis via endosonographically guided fine-needle aspiration of foregut/hindgut cyst is accurate and less traumatic than surgical biopsies. Diagn. Cytopathol. 2007;35:43-46. @ 2006 Wiley-Liss, Inc.

Key Words: foregut cyst; tailgut cyst; fine-needle aspiration; FNA

Alimentary duplication cysts are infrequent anomalies in the development of the digestive tract from the embryonic foregut, midgut, hindgut, and tailgut. The embryonic foregut develops into organs of both the respiratory and digestive tract, including pharynx, trachea, bronchi, esophagus, stomach, proximal duodenum, and hepatobiliary system. The midgut extends from the distal duodenum to the mid-transverse colon and is supplied by the superior mesenteric artery. The hindgut extends from the distal transverse colon through the rectum, deriving its blood supply from the inferior mesenteric artery. The tailgut is the

Department of Pathology, University of Michigan Medical Center, Ann Arbor, Michigan

*Correspondence to: Yijun Pang, M.D., Ph.D., Department of Pathology, University of Michigan, 1500 E. Medical Center Drive, Room 2G332, Box 0054, Ann Arbor, MI 48109.

E-mail: ypang@med.umich.edu

Received 23 May 2006; Accepted 25 August 2006

DOI 10.1002/dc.20573

Published online in Wiley InterScience (www.interscience.wiley.com). postrectal portion of the embryonic digestive tract that regresses with development.

Alimentary cysts, especially those in unusual locations, can present difficult challenges for clinical diagnosis. Frequently, their cystic nature is not appreciated upon radiologic evaluation. In one series of 10 foregut cysts, CT scan mischaracterized $70 \%$ as solid masses. ${ }^{1}$ In another series of 20 benign mediastinal cysts, ultrasonography provided increased sensitivity in revealing their cystic nature: a clear cystic pattern of a well-defined wall with an anechoic center was observed in 12 cases. Hypoechoic patterns were also observed, probably resulting from high viscosity of the proteinaceous cyst contents, which would similarly result in mass-like density on CT scan. ${ }^{2}$ In solid-appearing lesions, a neoplasm should not be excluded a priori. Moreover, a proportion of neoplasms are predominantly cystic. Thus, tissue diagnosis is warranted in both these presentations.

We describe a foregut cyst at the lesser curvature of the stomach and a retrorectal hind/tailgut cyst that presented as masses in adults. Diagnoses were ultimately provided by endosonographically guided fine needle aspiration.

\section{Case Descriptions \\ Case 1}

A 40-year-old man presented for evaluation of dyspnea upon exertion and right upper quadrant discomfort of 6 mo duration. His abdominal pain was not related temporally to eating. He reported no changes in bowel habits and was not jaundiced. Physical examination and laboratory studies (CBC, amylase, lipase, and comprehensive metabolic panel) were unremarkable. No tumor marker chemistry studies were obtained. A CT scan of the abdomen revealed a $6.1 \times 5.2 \mathrm{~cm}^{2}$ slightly hypodense and homogeneous soft tissue mass in the epigastric region. This was located in the gastroduodenal space posterior to the 


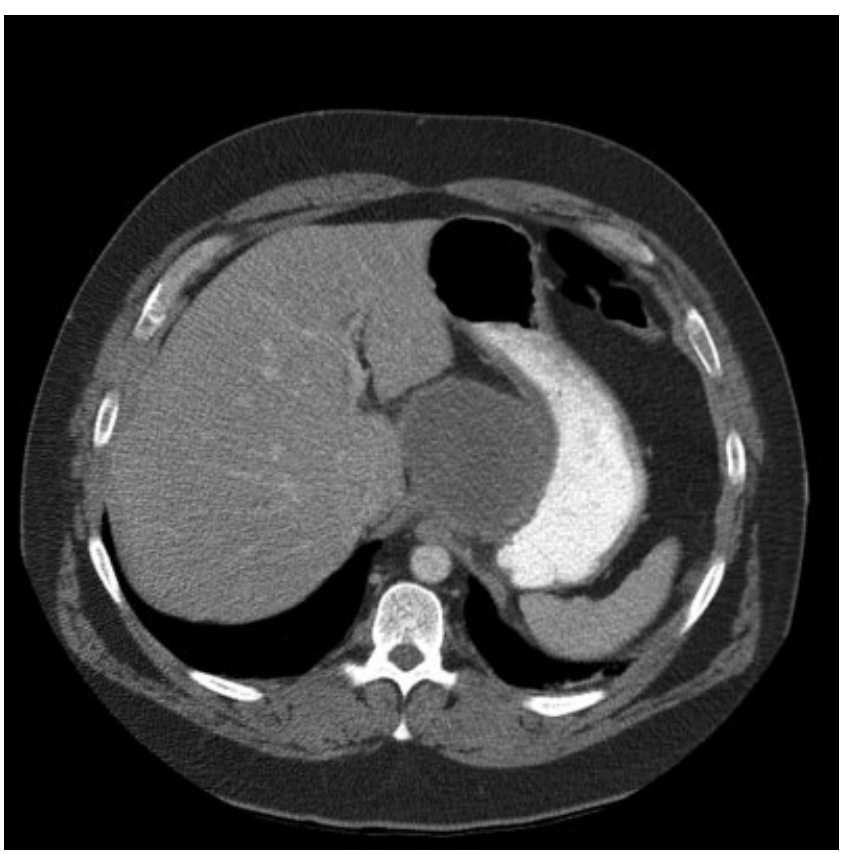

Fig. 1. Abdominal CT scan of patient one with a $6.1 \times 5.2 \mathrm{~cm}^{2}$ hypodense, homogeneous mass adjacent to posterior stomach.

lateral segment of the left hepatic lobe, slightly indenting the lesser curvature of the stomach and distal esophagus at the posterior gastroesophageal junction (Fig. 1). The radiological differential diagnosis included a smooth muscle/gastrointestinal stromal tumor, nerve sheath tumor, and enlarged lymph nodes.

An endoscopic ultrasound examination was performed by the gastroenterologist, revealing a subepithelial $5.4 \mathrm{~cm}$ hypoechoic lesion, which was in continuity with the muscularis propria. Material from the lesion was obtained via transgastric fine-needle aspiration with three passes of a 22-gauge needle. Three air-dried smears were stained with a Diff-Quick method and evaluated for adequacy on site. The slides demonstrated a hypocellular specimen with abundant mucin admixed with degenerative cellular debris. Many macrophages, and rare clusters of columnar epithelial cells were present. The epithelial cells had a benign appearance, characterized by round and oval regular nuclei with smooth chromatin and low nuclear to cytoplasmic ratios (Fig. 2). No spindled cells of mesenchymal differentiation were noted. Although epitheliod stromal tumors may occur, the cells in this case were differentiated as epithelial cells rather than epitheliod in morphology. Thus the differential diagnosis of GIST was excluded on site. Three alcohol-fixed smears were processed with Papanicolaou stain. The columnar epithelial cells were also present on these slides, exhibiting terminal bars and apical cilia. The morphology resembled benign bronchial epithelium. The diagnosis of congenital foregut cyst was

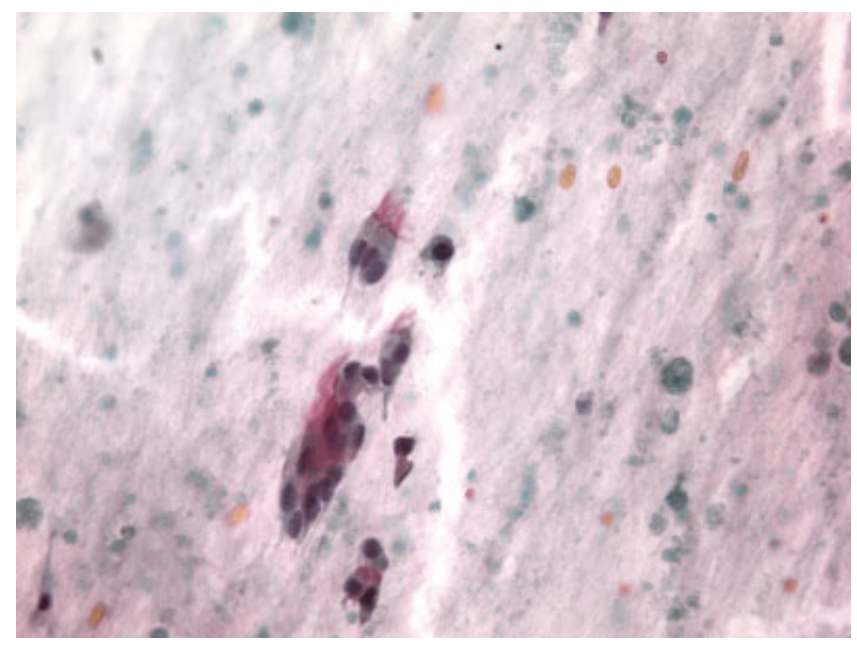

Fig. 2. Fine needle aspirate $(\times 400$, Papanicolaou stain $)$ from patient one showing degenerated cells and numerous benign ciliated epithelial cells with terminal bars. [Color figure can be viewed in the online issue, which is available at www.interscience.wiley.com.]

made based upon the findings. The patient experienced abdominal pain following the fine-needle aspiration, probably as a consequence of infection following inadequate antibiotic prophylaxis. He was treated with antibiotics and has not elected surgical resection.

\section{Case 2}

A 49-year-old woman was referred to our institution for evaluation of a perirectal mass noted upon digital rectal examination. Upon endosonography, a submucosal $2.5 \times$ $2.3 \mathrm{~cm}^{2}$ hypoechoic mass with few internal septae was observed in the retrorectal space anterior to the sacrum. The lesion involved the serosa and appeared to have both solid and cystic components with through transmission. Dark, viscous material from the lesion was obtained via transrectal fine-needle aspiration with six passes of a 22gauge needle. Six air-dried smears were stained with a Diff-Quick method and evaluated for adequacy on site. The slides demonstrated a cellular specimen with numerous degenerated squamous cells with regular small nuclei and moderate amount of cytoplasm. Cellular debris and proteinaceous material were present in the background. On one of the slides, a few groups of glandular cells in a single layer and organized pattern were seen. Goblet cells were identified among the benign appearing glandular cells. Ultimately, a few cells exhibited terminal plates and cilia (Fig. 3). The same components of the specimen were revealed on the alcohol-fixed smears stained with a Papanicolaou method. Smooth chromatin was appreciated in the well-preserved squamous cells as well as in the ciliated glandular cells. The debris that mimicked necrosis 

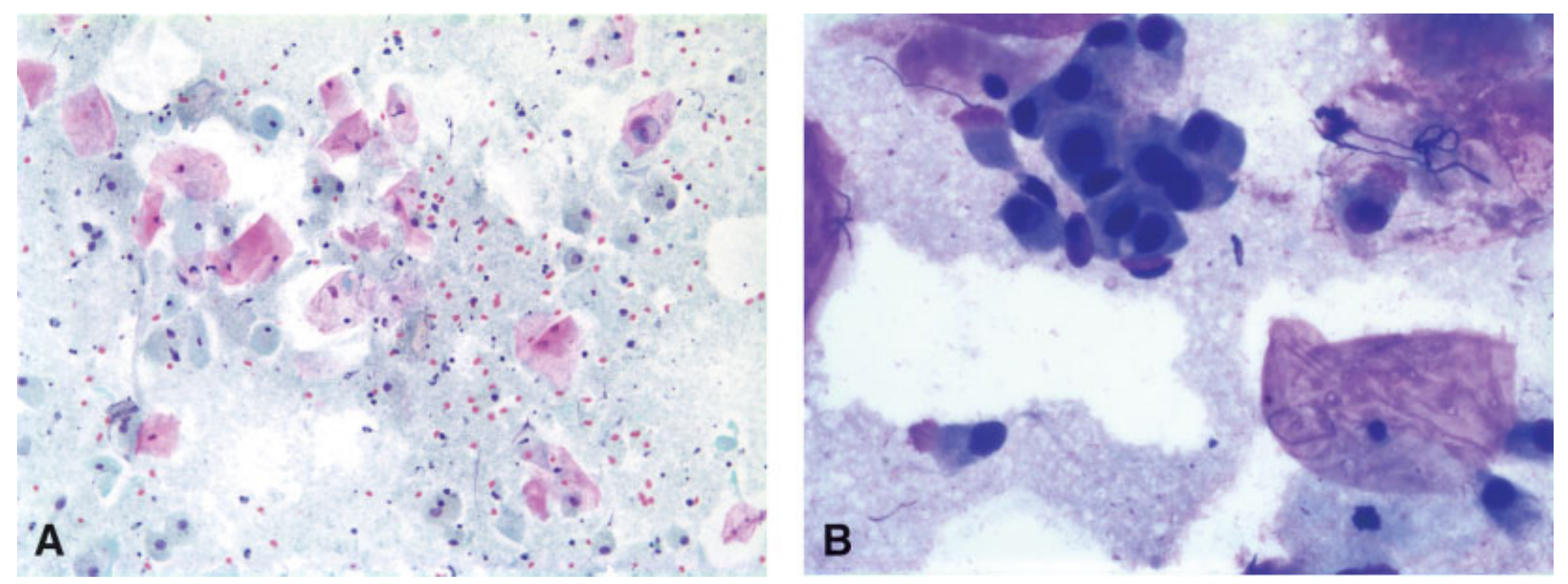

Fig. 3. Fine needle aspirate from patient two showing debris, macrophages, and numerous benign squamous cells (A: 400×, Papanicolaou stain). Occasional benign ciliated columnar cells were observed (B: $600 \times$, Diff-Quik). [Color figure can be viewed in the online issue, which is available at www.interscience.wiley.com.]

was excluded from being malignant with the identification of ciliated cells and lack of atypia in cellular component. The diagnosis of a congenital tailgut cyst was made. The patient was reassured, did not elect resection, and was subsequently lost to follow-up.

\section{Discussion}

Two closely related types of foregut-derived cysts are bronchogenic cysts and alimentary duplication cysts. Bronchogenic cysts contain pseudostratified columnar or cuboidal ciliated epithelium, seromucinous glands, smooth muscle, and cartilage. These are not uncommon in the mediastinum of children and adults, accounting for $\sim 20 \%$ of mediastinal masses and presenting with symptoms of airway compression as cough or dyspnea. ${ }^{3}$ Subdiaphragmatic bronchogenic cysts, though, are extremely unusual; fewer than 50 cases have been reported. ${ }^{4}$ In contrast to the bronchogenic cyst, alimentary duplication cysts contain digestive epithelium in lieu of respiratory epithelium and do not contain cartilage. Although these may involve the foregut, they are most prevalent in the small intestine. Gastric duplications are rare, accounting for less than $10 \%$ of alimentary duplication cysts. ${ }^{5}$ Most gastric duplication cysts are detected in children, with a slight female predominance. Presenting symptoms include mass, vomiting, or pain; but they may also be asymptomatic. Gastric duplication cysts tend to located along the greater curvature of the stomach, although other locations including the lesser curvature have been reported.

Similarly, hindgut and tailgut cysts affecting the anorectum are uncommon, accounting for less than 3\% of alimentary duplication cysts. ${ }^{5}$ These cysts may be asymptomatic or cause mass-related symptoms such as pain or constipation. Hind or tailgut cysts may also present as a sinus, fistula or an incidental mass detected upon rectal examination. Unlike gastric duplication cysts, hindgut and tailgut cysts are seen in a wide range of patient ages, being most prevalent in adult women. Hindgut cysts are usually unilocular, whereas tailgut cysts are usually multilocular. Both contain only alimentary tract tissues and are retrorectal/precoccygeal in location, distinguishing them from sacral teratomas, which also contain nondigestive tissues and are typically postcoccygeal. Hind/tailgut cysts may contain squamous, columnar (mucin secreting or ciliated), or transitional epithelium. Although ciliated epithelium is not observed in the adult digestive tract, it lines the embryonic alimentary tract during development, and thus represents a remnant in these cysts. ${ }^{6}$

Several mechanistic theories have been proposed to explain the development of alimentary cysts. They may be derived from pinched off diverticula, fusion of longitudinal enteric folds, or errors in recanalization of the developing digestive tract. ${ }^{7}$ Alternatively, ciliated foregut cysts may be remnants of a caudal portion of the tracheolaryngeal outpouching that remains attached to the alimentary portion of the developing foregut rather than separating with the tracheolaryngeal tube. ${ }^{8}$ Traction from endodermal-ectodermal adhesions (neurenteric bands) may also contribute to the evolution of alimentary cysts, especially hind/tailgut cysts and those associated with vertebral malformations. ${ }^{6}$

Traditionally, pathologic diagnosis of foregut and hind/ tailgut cysts was provided upon resection. More recently, however, two gastric foregut cysts and one tailgut cyst have been diagnosed based upon cytology.,10 Foregut/ hindgut cysts can be lined by variety of epithelial cell types, most often, columnar/cuboidal glandular cells with features of bronchial epithelium, gastrointestinal epithelium and squamous cells. The long term persisting nature of the cysts usually results in accumulation of mucinous 
material with degenerated cellular debris, which may be misleading for necrosis and raise the suspicions for malignancy. With a careful search, there will be no marked atypia to support a diagnosis of malignancy. Additionally. in a subdiaphragmatic location, ciliated respiratory-type epithelial cells would not normally be expected; hence, the presence of intact ciliated cells, in a background of cyst contents including annucleate cell fragments, macrophages and proteinaceous material with no evidence of malignancy, are the major cytologic criteria for the diagnosis of foregut duplication cysts. ${ }^{1}$ A few cases of gastric duplication cysts with ciliated respiratory-type epithelium have been described ${ }^{8,11-14}$; therefore, one can not readily cytologically distinguish a subdiaphragmatic bronchogenic cyst from a gastric duplication cyst. We thus preferred the more encompassing term foregut cyst for cytologic diagnosis in our first case.

Endosonographically guided fine-needle aspiration is generally considered to be an accurate and reliable procedure to obtain cytopathology specimens. It is minimally invasive. One of our patients, however, developed a postprocedural infection. Inadequate antibiotic prophylaxis likely contributed to this complication. In recent studies examining the safety and utility of this procedure, only two other infections have been reported. Both occurred in patients without antibiotic prophylaxis. ${ }^{2,15}$

Surgical resection has been the traditional therapy for foregut and hind/tailgut cysts; however, malignant components have only rarely been reported these developmental anomalies. $^{6,10,16-19}$ More recently, a gastric duplication cyst has been therapeutically aspirated via endoscopy. ${ }^{20}$ It is conceivable that current endoscopic ultrasonographic and cytologic diagnosis may allow advances in clinical management, including observation, to postpone or altogether avoid a surgical procedure in the absence of unrelenting symptoms.

\section{References}

1. Eloubeidi MA, Cohn M, Cerfolio RJ, Chhieng DC, Jhala N, Jhala D, Eltoum IA. Endoscopic ultrasound-guided fine-needle aspiration in the diagnosis of foregut duplication cysts: The value of demonstrating detached ciliary tufts in cyst fluid. Cancer Cytopathol 2004;102:253258 .
2. Wildi SM, Hoda RS, Fickling W, Schmulewitz N, Varadarajulu S, Roberts SS, Ferguson B, Hoffman BJ, Hawes RH, Wallace MB. Diagnosis of benign cysts of the mediastinum: The role and risks of EUS and FNA. Gastrointest Endosc 2003;58:362-368.

3. Sirivella S, Ford WB, Zikria EA, Miller WH, Samadani SR, Sullivan ME. Foregut cysts of the mediastinum. Results in 20 consecutively treated cases. J Thorac Cardiovasc Surg 1985;90:776-782.

4. Liang MK, Yee HT, Song JW, Marks JL. Subdiaphragmatic bronchogenic cysts: A comprehensive review of the literature. Am Surg 2005;71:1034-1041.

5. Bower RJ, Sieber WK, Kieseweller WB. Alimentary tract duplications in children. Ann Surg 1978;188:669-674.

6. Hjermstad BM, Helwig EB. Tailgut cysts. Report of 53 cases. Am J Clin Pathol 1988;89:139-147.

7. Bremmer JL. Diverticula and duplications of the intestinal tract. Arch Pathol Lab Med 1944;38:132-140.

8. Gensler S, Seidenberg B, Rifkin H, Rubinstein BM. Ciliated lined intramural cyst of the stomach: Case report and suggested embryogenesis. Ann Surg 1966;163:954-956.

9. Ponder TB, Collins BT. Fine needle aspiration biopsy of gastric duplication cysts with endoscopic ultrasound guidance. Acta Cytol 2003;47:571-574.

10. Umar T, Mikel JJ, Poller DN. Carcinoma arising in a tailgut cyst diagnosed on fine needle aspiration (FNA) cytology. Cytopathology 2000;11:129-132.

11. Shireman PK. Intramural cyst of the stomach. Hum Pathol 1987;18: 857,858 .

12. Laraja RD, Rothenberg RE, Chapman J, Imran-Ul-Haq, Sabatini MT. Foregut duplication cyst: A report of a case. Am Surg 1995;61:840, 841.

13. Takahara T, Torigoe $\mathrm{T}$, Haga H, Yoshida H, Takeshima S, Sano S, Ishii Y, Furuya T, Nakamura E, Ishikawa M. Gastric duplication cyst: Evaluation by endoscopic ultrasonography and magnetic resonance imaging. J Gastroenterol 1996;31:420-424.

14. Kim D-H, Kim J-S, Nam ES, Shin HS. Foregut duplication cyst of the stomach. Pathol Int 2000;50:142-145.

15. Fazel A, Moezardalan K, Varadarajulu S, Drananov P, Eloubeidi MA. The utility and the safety of EUS-guided FNA in the evaluation of duplication cysts. Gastrointest Endosc 2005;62:575-580.

16. Coit DG, Mies C. Adenocarcinoma arising within a gastric duplication cyst. J Surg Oncol 1992;50:274-277.

17. Mayo MW, Jr, McKee EE, Anderson RM. Carcinoma arising in reduplication of the stomach: A case report. Ann Surg 1955;141:550555.

18. Aleodor A, Klimstra DS. Adenocarcinoma arising in a tailgut cyst with prominent meningothelial proliferation and thyroid tissue: Case report and review of the literature. Virchows Arch 2005;446:316-321.

19. Krivokapic Z, Dimitrijevic I, Barisic G, Markovic V, Krstic M. Adenosquamous carcinoma arising within a retrorectal tailgut cyst: Report of a case. World J Gastroenterol 2005;11:6225-6227.

20. Ferrari AP, Van Dam J, Carr-Locke DL. Endoscopic needle aspiration of a gastric duplication cyst. Endoscopy 1995;27:270-272. 